\title{
Using current pulses to control the intermediate conductance states in hafnium oxide-based RRAM devices
}

\author{
Héctor García, Óscar G. Ossorio, Salvador Dueñas, and Helena Castán
}

\begin{abstract}
Artificial synaptic devices used in neuromorphic systems need a high number of reachable conductance levels. Resistive switching devices are promising candidates to implement these devices due to their reachable conductance levels. In this work, we have used $\mathrm{TiN} / \mathrm{Ti} / \mathrm{HfO}_{2} / \mathrm{W}$ capacitors to study the control of the intermediate conductance states using current pulses instead of the usual voltage pulses. Unlike the use of voltage pulses, in this case we can control the HRS to LRS transition (potentiation characteristic). The characteristic is clearly linear when applying current pulses with linearly increasing amplitudes. The potentiation characteristic is not affected by the pulse length, even for lengths lower than $1 \mu \mathrm{s}$. In terms of peripheral circuitry, it is desirable to use pulses with identical amplitudes, but in this case no accumulative behavior is observed, and one current pulse is enough to carry the device to the final conductance state achieved for the amplitude used. Finally, it is not possible to control the HRS to LRS transition (depression characteristic) using current pulses due to the abrupt reset transition. However, this transition can be well controlled using voltage pulses.
\end{abstract}

Index Terms-Resistive switching memory (RRAM), synaptic devices, multilevel storage.

\section{INTRODUCTION}

$\mathrm{T}$ HE resistive switching (RS) phenomena is used in resistive random-access memories (RRAMs) to store binary information [1-2]. In these devices, a conductive filament (CF) formed between a top electrode and a bottom electrode by reversible dielectric breakdown (set operation) results in the low resistance state (LRS). A high resistance state (HRS) is obtained in the reset operation, when the conductive filament is dissolved. However, the thickness of the conductive filament can be controlled in different ways obtaining not only two different conductance states, but also a continuum number of conductance values [3].

A high number of reachable conductance levels is a required property to implement analog synapses. Analog synapses are used in neuromorphic systems, which are inspired in human brain and other biological systems [4-5]. The synaptic weight between two neurons is adjusted by the ionic flow through them. Similar to biological synapses, the conductance value of

This work was funded by the Spanish Ministry of Economy and Competitiveness and the FEDER program through project TEC2017-84321C4-2-R. The authors are with the Department of Electronics, University of a RRAM device can be incrementally adjusted by controlling the charge through it. It is believed that the adaptation of synaptic weights enables the biological systems to learn and function properly [6].

In a previous work, we studied the control of the intermediate conductance states in hafnium oxide $\left(\mathrm{HfO}_{2}\right)$ based metalinsulator-metal (MIM) capacitors, by applying voltage pulses [7]. In the case of the potentiation characteristic, we obtained a bad behavior due to the abrupt set characteristic. In this work, we have studied the control of the conductance states in the same samples, but using current pulses instead of voltage pulses. A linear potentiation characteristic is obtained, although different pulses amplitudes are needed. However, the use of current pulses does not allow obtaining a linear depression characteristic.

\section{EXPERIMENT SECTION}

The devices used in this work are TiN/Ti/10-nm $\mathrm{HfO}_{2} / \mathrm{W}$ MIM capacitors. The high-k dielectric was deposited by the atomic layer deposition (ALD) technique at $225{ }^{\circ} \mathrm{C}$ using TDMAH and water as hafnium and oxygen precursors respectively. Nitrogen was used as carrier and purge gas. The bottom electrode consists of a $200 \mathrm{~nm} \mathrm{~W}$ layer, and the top electrode consists of a $200 \mathrm{~nm}$ TiN layer and a $10 \mathrm{~nm}$ Ti layer. Metal electrodes were deposited by magnetron sputtering.

An HP 4155B Semiconductor Parameter Analyzer was used to perform the voltage and the current measurements. In order to perform the pulsed measurements, we used an Agilent 33500B Series waveform generator to apply voltage pulses. The voltage pulses are converted to current pulses using a homemade voltage to current converter. All the measurements were carried out at room temperature and the equipment were computer controlled.

\section{RESULTS AND DISCUSSION}

The resistive switching mechanism in our RRAM devices is valence change memory effect (VCM), as it was studied in a previous work [8]. The conductive path of the switching layer is formed due to oxygen ion migration and formation of

Valladolid, Paseo de Belén 15, 47011 Valladolid (Spain). (e-mail: hecgar@ele.uva.es; ogonoss@ribera.tel.uva.es; sduenas@ele.uva.es; helena@ele.uva.es;). 


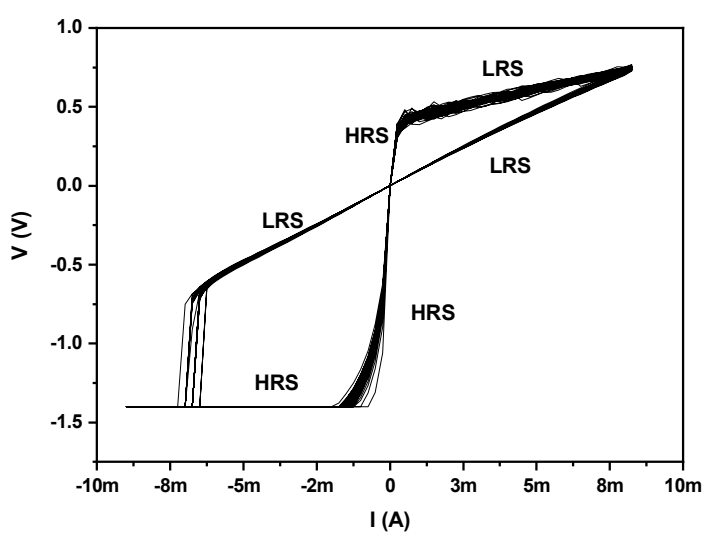

Fig. 1. Several V-I resistive switching loops showing set and reset processes.

conductive filaments [9]. As we are interested in the behavior of the sample when current pulses are applied, we have represented the voltage-current (V-I) characteristic instead of the current-voltage (I-V) usual characteristic. Fig. 1 shows 50 consecutive V-I loops. We can observe a bipolar RS behavior. When applying current instead of voltage, we have to use a voltage compliance in the reset transition; otherwise, irreversible oxide breakdown will take place due to a quick increase in the power dissipated by the structure.

Fig. 2(a) shows the voltage-current characteristics when increasing the maximum current value from one loop to the next one. This figure clearly shows there exist intermediate conductance values in the structures. Fig. 2(b) shows the conductance values for the different maximum currents applied, obtained from Fig. 2(a). The CF filament is formed at low currents so we are mainly observing an increase in its thickness. The relationship between the conductance value and the maximum current is linear, so a way to control the conductance values (synaptic weight in the neuroscience) is achieved for the HRS to the LRS transition, which is known as potentiation characteristic in neuromorphic systems. The depression characteristic (the LRS to the HRS transition) cannot be controlled by varying the maximum negative current applied due to the abrupt LRS to HRS transition. However, the depression characteristic is the one better controlled by applying voltage instead of current signals to the sample [7].

We have applied current pulses in order to observe the potentiation characteristic. The pulses length was $50 \mu \mathrm{s}$, and the pulses amplitude was linearly increased from $0 \mathrm{~mA}$ to $5.8 \mathrm{~mA}$. The sample was first carried out to the HRS and then, a total of 200 pulses were applied. The result has been represented in Fig. $3(a)$, where we can observe a continuum of intermediate conductance values linearly controlled by the applied pulses. However, a minimum current value of about $200 \mu \mathrm{A}$ should be applied in order to start increasing the synaptic weight (see dot line in Fig. 3(a)). We have also studied the depression characteristic applying 170 consecutive current pulses after carrying the sample to the LRS. We used the same pulse length and increased linearly the pulses amplitude from 0 ma to -8.5 $\mathrm{mA}$. In the case of the depression characteristic, no conductance a)
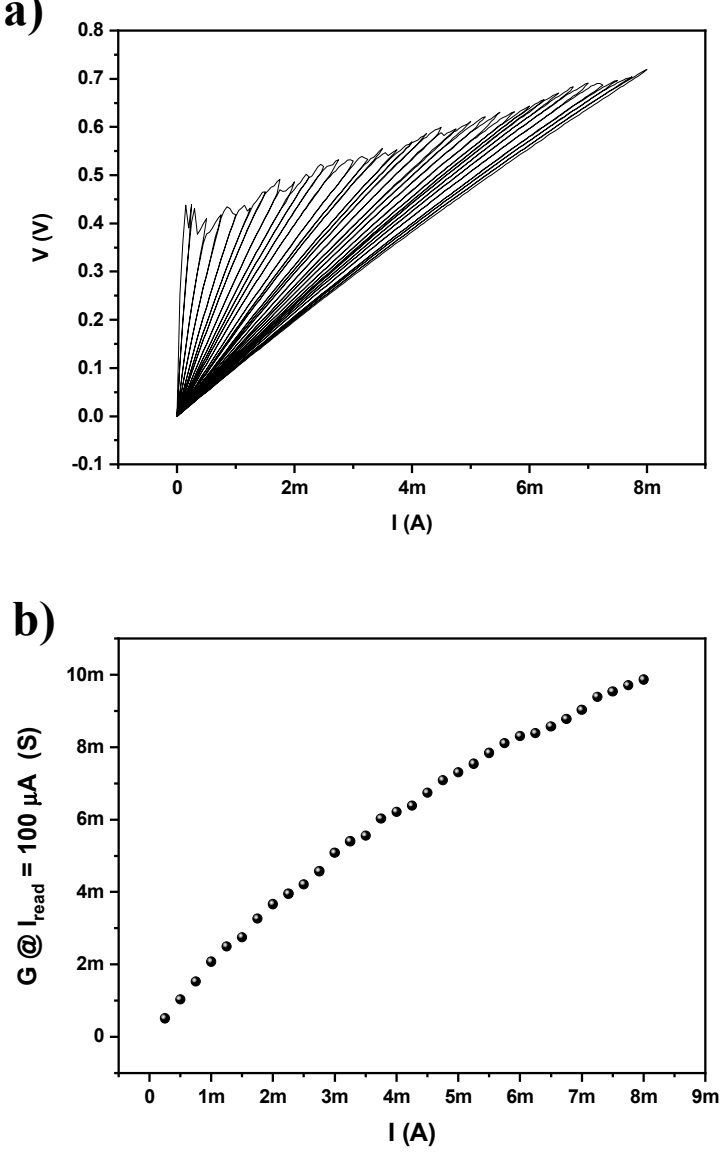

Fig. 2. Current-voltage characteristics when increasing the maximum current (a) and conductance values measured at $100 \mu \mathrm{A}$ for the different maximum currents applied.

changes are observed before reset transition takes place. At this point, a sudden change in the conductance values comes about. This behavior is the same observed in Fig. 1, so as we said above, the LRS to the HRS transition cannot be controlled by applying the current pulses. If voltage pulses are used to control this transition, the resistance value of the sample increases as the pulses are applied, so the current applied to the sample decreases for consecutive pulses, being able to obtain a linear depression characteristic.

When voltage instead of current pulses are applied to the samples, simulations demonstrated the final conductance value has a strong relationship with the maximum voltage and with the time that the pulse is applied [10]. We observed that when applying shorter voltage pulses to the samples a higher number of pulses were needed to obtain the same final synaptic weight. In order to check this trend when applying current pulses, we have applied three series of 50 pulses each series, after carrying out the sample to the HRS. The pulse length of each series was different, varying from $1 \mu \mathrm{s}$ to $50 \mu \mathrm{s}$, and the pulse amplitude was linearly increased from 0 to $5.8 \mathrm{~mA}$ in each series. We have represented the results obtained in Fig. 4. In this case, no significant differences are observed for different pulse lengths. It looks like the $\mathrm{CF}$ widening should be much faster than the lowest pulse length $(1 \mu \mathrm{s})$, as no differences are observed for 

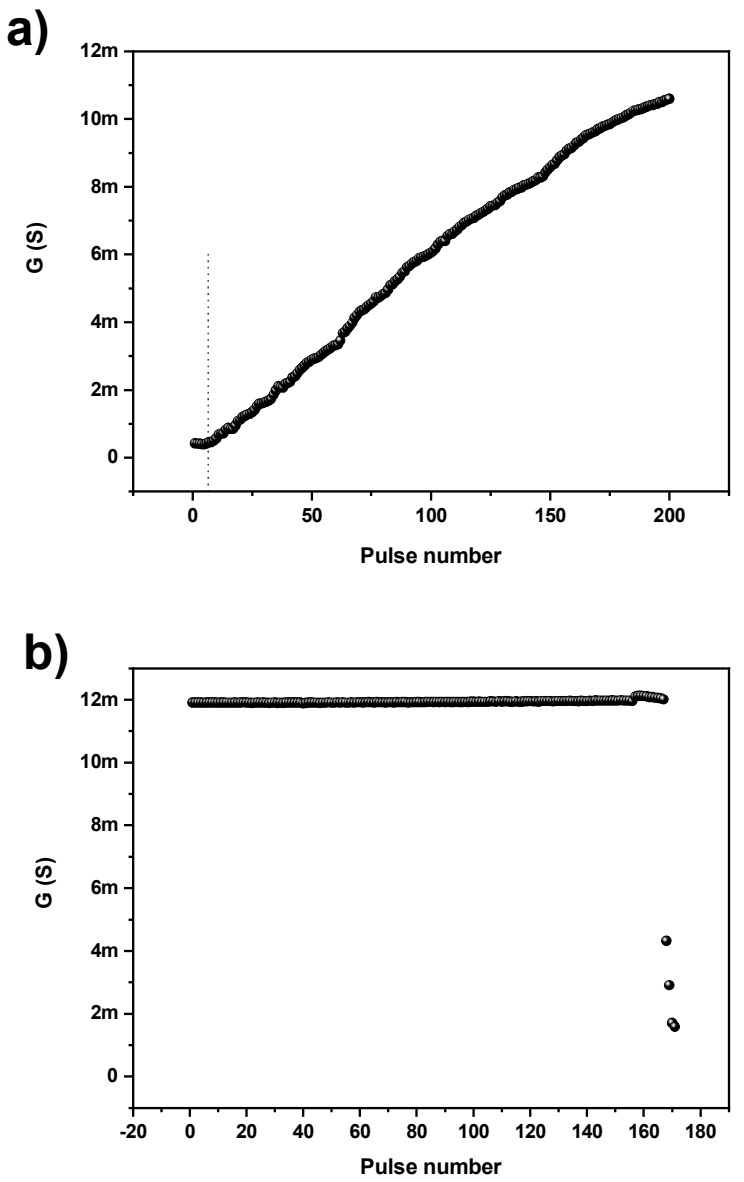

Fig. 3. Potentiation characteristic obtained by applying current pulses (50 $\mu \mathrm{s}$ length) linearly increasing the amplitude from 0 to $5.8 \mathrm{~mA}$ (a) and depression characteristic obtained by applying current pulses (50 $\mu$ s length) linearly increasing the amplitude from 0 to $-8.5 \mathrm{~mA}$ (b).

the different pulse lengths.

We have applied consecutive pulses with different amplitudes so far. However, this has a disadvantage in terms of peripheral circuitry complexity. The peripheral circuitry used to apply the pulses would be easier if all the applied pulses had the same length and amplitude. So we studied the effect of applying consecutive pulses, but keeping the current amplitude constant. We have used three different current amplitudes $(1,2,3$, and 4 $\mathrm{mA}$ ), as shown in Fig. 5. In this measurements, we have kept constant the pulse length at $50 \mu \mathrm{s}$, although no significant differences were observed when varying this parameter. However, unlike the voltage pulses, the current pulses do not show an accumulative behavior: the first current pulse increases the conductance value to the final value expected for our V-I measurements. The reason for this behavior is similar to the one commented above: when voltage pulses are applied, the resistance values decreases in this case as the conductive filament thickness increases, so if we apply voltage pulses with the same amplitude is equivalent to apply current pulses with an increasing amplitude, showing the voltage pulses an accumulative behavior. However, we are here using current pulses with the same amplitude. This argument can be used to explain the results observed in Fig. 4, as applying pulses with

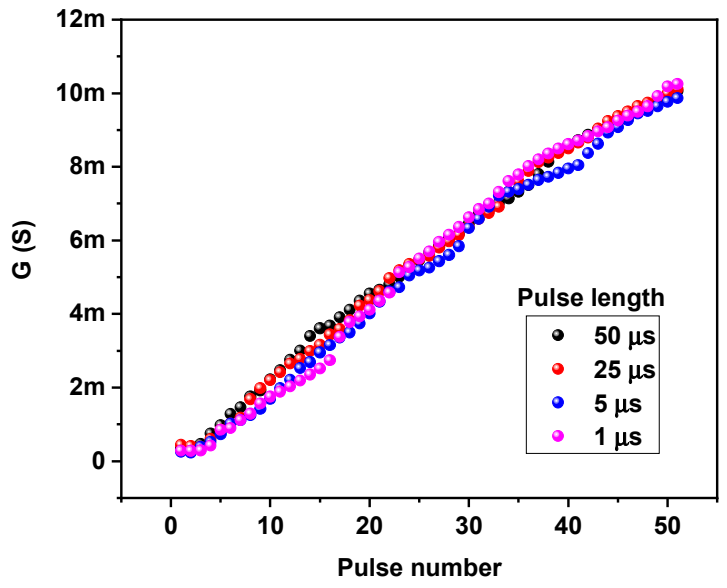

Fig. 4. Potentiation characteristic obtained by applying current pulses with different lengths, and linearly increasing the amplitude from 0 to $5.8 \mu \mathrm{A}$.

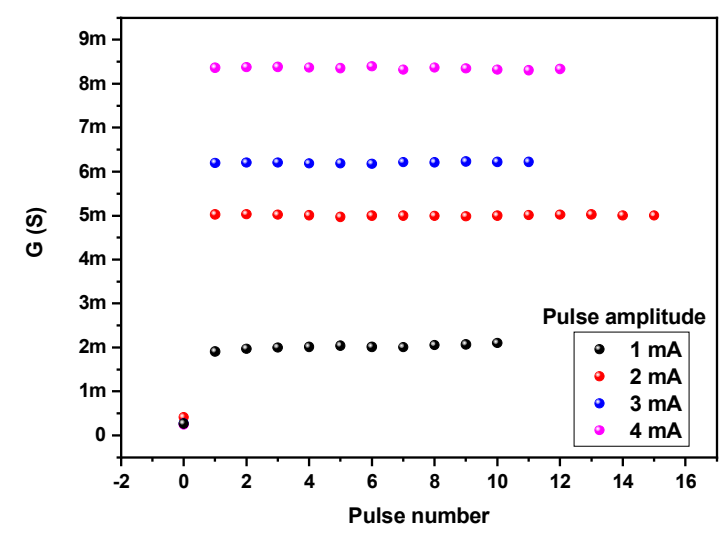

Fig. 5. Potentiation characteristic obtained by applying current pulses with the same amplitude.

higher length is similar to apply several pulses with lower length but the same amplitude.

We have finally studied the possibility of using very short pulses to be able to use the same pulse amplitude to control the HRS to LRS transition. We have used three pulses with different lengths $(1 \mu \mathrm{s}, 500 \mathrm{~ns}$ and $50 \mathrm{~ns})$ and the same amplitude $(4.8 \mathrm{~mA})$. The sample was first carried to the HRS, and, after applying only one pulse, we performed a currentvoltage measurement. We have represented the results obtained in Fig. 6. The black line corresponds to a measure after carrying the sample to the LRS. The blue and magenta lines correspond to a measure after applying pulses with $1 \mu$ s and $500 \mathrm{~ns}$ length. The same intermediate conductance state is achieved in both cases. However, when applying the $50 \mathrm{~ns}$ length pulse, we reach an intermediate state with a lower conductance value. However, we have observed that after applying only three or even two of these pulses, we reach the intermediate state obtained for the 1 $\mu$ s and 500 ns length pulses. This means once the conductive filament has been formed, the widening of this filament is so fast that a pulse as short as $50 \mathrm{~ns}$ widens the filament to its final state. 


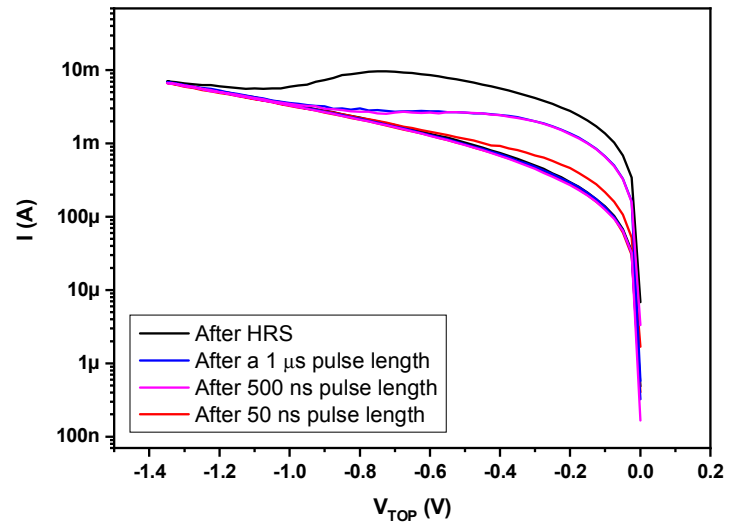

Fig. 6. Current-voltage characteristics after applying a single current pulse with different length.

\section{CONCLUSIONS}

Resistive switching effect is observed in $\mathrm{HfO}_{2}$-based MIM capacitors, and they also shows intermediate conductance states, so these devices are candidates for their use as artificial synaptic devices. Measurements applying current instead of voltage showed that increasing the maximum current applied to the capacitors is a way to reach the intermediate conductance states in the case of the HRS to LRS transition. However, we are no able to reach the intermediate conductance states in the case of the LRS to HRS transition due to the abrupt reset transition. We focused our attention on the potentiation characteristic. We studied the application of current pulses to change the synaptic weight, a more realistic method than applying current sweeps. A linear potentiation characteristic is obtained when applying current pulses with linearly increasing amplitude. We are really controlling the thickness of the conductive filament. However, the pulse length does not affect the characteristic, even for lengths shorter than $1 \mu \mathrm{s}$. The widening of the conductive filament is too fast to be able to control it by pulse length instead of pulse amplitude. In terms of peripheral circuitry simplicity, it is desirable to apply identical pulses (same length and same amplitude). If we apply consecutive identical pulses, no accumulative behavior is observed: it is enough the applying of one only pulse to carry the sample to the final conductance state achieved for the amplitude applied. The reason is the same, the fast widening of the conductive filament. In conclusion, applying current pulses is a correct method to control the potentiation characteristic, which could be combined with the voltage pulses to control the depression characteristic.

\section{ACKNOWLEDGMENT}

Authors would like to acknowledge Prof. Campabadal group from the Institute of Microelectronics of Barcelona (IMBCNM-CSIC, Spain) for providing the samples of this study.

\section{REFERENCES}

[1] D. Ielmini, "Resistive switching memories based on metal oxides: mechanisms, reliability and scaling," Semicond. Sci. Technol., vol. 31, no. 6, pp. 063002 , Jun. 2016.

[2] T.-C. Chang, K.-C. Chang, T.-M. Tsai, T.-J. Chu, and S. M. Sze, "Resistance Random Access memory," Mater. Today, vol. 19, no. 5, pp. 254.264, Jun. 2016.

[3] H. Castán, S. Dueñas, H. García, O. G. Ossorio, L. A. Domínguez, B. Shaelices, E. Miranda, M. B. González, and, F. Campabadal, "Analysis and control of the intermediate memory states of RRAM devices by means of admittance parameters," J. Appl. Phys., vol. 124, no. 15,pp. 152101, Oct. 2018.

[4] D. Ielmini, "Brain-inspired computing with resistive switching memory (RRAM): Devices, synapses and neural networks," Microelectron. Eng., vol. 190, pp. 44-53, Apr. 2018.

[5] X. Hong, S. J. Loy, P. A. Dananjaya, F. Tan, C. Ng, and W. Lew, "Oxidebased RRAM materials for neuromorphic computing," J. Mater. Sci, vol. 53, no. 12, pp. 8720-8746, Jun. 2018.

[6] S. Song, K. D. Miller, and L. F. Abbott, "Competitive Hebbian learning through spike-timing dependent synaptic plasticity," Nature Neurosci., vol. 3, no.9, pp. 919-926, Sept. 2000.

[7] H. García, O. G. Ossorio, S. Dueñas, and H. Castán, Microelectron. Eng., vol. 215, pp. 110984, Jul. 2019.

[8] G. González-Cordero, M. B. González, H. García, F. Campabadal, S. Dueñas, H. Castán, F. Jiménez-Molinos, and J. B. Roldán, “A physically based-model for resistive memories including a detailed temperature and variability description," Microelectron. Eng., vol. 178, pp. 26-29, Jun. 2017.

[9] R. Wasser, R. Dittmann, G. Staikov, andK. Szot, "Redox-based resistive switching memories - nanoionics mechanisms, prospects, and challenges," Adv. Mater., vol. 21, no. 25-26, Jul. 2009.

[10] Y. Li, M. Zhang, S. Long, J. Teng, Q. Liu, H. Lv, E. Miranda, J. Suñé, and M. Liu, "Investigation on the conductive Filament Growth Dynamics in Resistive Switching Memory via a Universal Montecarlo Simulator," Sci. Rep., vol. 7, pp. 11204, Sep. 2017. 